\title{
Overview in Invasive Lobular Carcinoma in Medical Oncology Department - Algeria
}

\author{
*Selma Sakhri \\ Department of Médical oncology, Algeria Hospital, Africa
}

Submission: July 04, 2017; Published: July 17, 2017

"Correspondence Address: Selma Sakhri, Department of Médical oncology, Algeria Hospital, algeria 10, rue docteur saadane, Algeria, Africa, Email: sakhri_selma@yahoo.fr

\section{Background}

Invasive lobular cancers (ILC) constitute 5\%-15\% of all invasive breast tumors, less common than invasive ductal carcinoma (IDC), and appear to have a distinct biology. The aim of our study is to determine the ILC biological profile collected in the department of Medical Oncology, Algeria.

\section{Methods}

We recorded the cases of 73 patients with ILC between January 2010 and January 2012. The aim of this study is to evaluate the clinical pathological response, and molecular profiling using RE, RP, KI67, HER2, and the follow up between (2012-2014).

\section{Results}

The average age of patients with ILC is 52 years, $40 \%$ women have postmenopausal status, all patients underwent mammography, diagnosed by a core needle biopsy, the commonest stage at presentation was stage III (42\%). 10 patients $(13 \%)$ underwent a breast conserving surgery, and $87 \%$ a mastectomy. The majority of cases were histologic grade II (SBR II), 50\% of the tumors were estrogen and progesterone receptor positive, Her 2 neu was reported to be negative in $60 \%$. The treatment consisted on: neoadjuvant chemotherapy in 28 cases (38\%), adjuvant chemotherapy in 42 cases $(57 \%)$, following by radiotherapy, and hormonal therapy (HT) was given to 47 patients (64\%), and target therapy to $40 \%$ the follow up after 2 years, $12 \%$ had metastatic or loco regional recurrence, and , $88 \%$ of the cases remain in complete remission.

\section{Discussion}

In our studies we found that the median age of ILC was 52 years, different when we compare this with the IDC (infiltrating ductal carcinoma). Fisher et al. [1] reported that lobular carcinoma exhibited a gradual increase in incidence with increasing age, with the highest incidence observed among patients aged $>70$ years. ILCs exhibit certain particularities compared to IDCs, particularly regarding metastatic behavior, however, the behavior of these breast tumors in women (aged $>52$ years) has not been clearly determined, partly due to their lower incidence. Therefore, we aimed to investigate certain clinico pathological and biological parameters and compare the results with those obtained from women (aged 3152 years) with the same type of tumor. We observed that the ILCs of patients aged $>52$ years tended to be of larger size, $6 \mathrm{~cm}$. There were no significant differences in the remaining analyzed parameters or in the incidence of recur $\neg$ rence and mortality between the two age subgroups.

As regards tumor size, our results were consistent with those reported by other group, indicating that tumor size increases with age and the increase is more significant after the age of 50 years [2]. We observed that the characteristics and the clinical behavior of breast carcinomas in women aged $>50$ years differs according to their biological profile. Tumors tended to be larger, with more frequent axillary node involvement and distant metastases when diagnosed by physical examination during a visit.

However, for tumors diagnosed through mammography and core needle biopsy, there were no signifi $\neg$ cant differences between women aged $>50$ and those aged 3152 years. Histological grade is higher in stage III, in our studies ILC is an entity in elderly women is associated with larger size, a higher number of metastatic lymph nodes, advanced clinical stage, higher HER2 negative rate, positive ER and PR; it is also associated with triple negative and HER2 subtypes [3-5]. ER, PR and Ki67, were not significantly different between the two age groups. Both local and systemic therapy for ILC differed according to biological profile; the number of patients who received adjuvant endocrine therapy with inhibitors aromatase is a better outcome than the others. 


\section{Cancer Therapy \& Oncology International Journal}

We noticed also that our patients did not have a bone recurrence because of the higher hormone receptor content. Recent data have shown that there is an improved response to the aromatase inhibitor letrozole compared with tamoxifène in ILCs [6] due to the genomic landscape of lobular carcinomas. After 2 years of follow up; $88 \%$ of the cases remain in complete remission.

\section{Conclusion}

Lobular carcinoma is an important breast cancer subtype with some clinical and biological characteristics, the disease is heterogeneous. A detailed assessment of the genomic landscape of a large cohort of ILCs with long-term follow-up and/or in the context of treatment resistance will no doubt be essential to moving forward with precision medicine for patients diagnosed with this tumor.

\section{Reference}

1. Fisher CJ, Egan MK, Smith P, Wicks K, Millis RR, et al. (1997) Histopathology of breast cancer in relation to age. Br J Cancer 75(4): 593596.

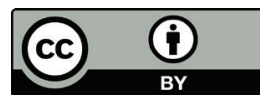

This work is licensed under Creative Commons Attribution 4.0 License

DOI: $10.19080 /$ CTOIJ.2017.06.555678
2. Schonberg MA, Marcantonio ER, Li D, Silliman RA, Ngo, et al. (2010) Breast cancer among the oldest old: tumor characteristics, treatment choices, and survival. J Clin Oncol 28: 20382045.

3. With lobular carcinoma in the BIG 1-98 trial (2012) In: Thirty-Fifth Annual CTRC-AACR San Antonio Breast Cancer Symposium, 72 San Antonio, Cancer Research p. S1-1.

4. Balducci L, Phillips DM (1998) Breast cancer in older women. Am Fam Physician 58(5): 11631172

5. Chen ST, Lai HW, Tseng HS, Chen LS, Kuo SJ (2011) Correlation of histologic grade with other clinicopathological parameters, intrinsic subtype, and patients' clinical outcome in Taiwanese women. Jpn J Clin Oncol 41(12): 13271335.

6. Metzger O, Giobbie-Hurder A, Mallon E, Viale G, Winer E, et al. (2015) Relative effectiveness of letrozole compared with tamoxifen for patients with Lobular Carcinoma in the BIG 1-98 Trial. J Clin Oncol 33(25): 2772-2779. 